\title{
Análise da eficiência relativa dos programas de pós-graduação acadêmicos em Administração, Contabilidade e Turismo
}

\author{
Ney Paulo Moreira, Nina Rosa da Silveira Cunba, \\ Marco Aurélio Marques Ferreira e Suely de Fátima Ramos Silveira
}

\section{Introdução}

O ensino superior veio a se estabelecer tardiamente no Brasil, se comparado a outros países latino-americanos (TeIXEIRA, 1989). Somente após a Proclamação da Independência é que se fundaram as primeiras faculdades; contudo, eram instituições independentes umas das outras e possuíam orientação profissional. Foram inspiradas nas grandes escolas francesas e seguiam esse modelo, ou seja, eram instituições mais voltadas ao ensino que à pesquisa (OLIVEN, 2002).

$\mathrm{Na}$ década de 1950, tornou-se evidente que o capital humano e o domínio do conhecimento científico-tecnológico eram condições indispensáveis ao desenvolvimento econômico-social (MACCARI, 2008). Teixeira (1989) afirma que as lacunas do ensino superior brasileiro vêm a acentuar-se depois da I Guerra Mundial, quando o desenvolvimento econômico do país passa a exigir a inclusão da pesquisa científica no ensino superior e na universidade, surgindo, então, a necessidade de uma escola pós-graduada. Por sua vez, Martins (2002) relata que vários fatores 
sociais, econômicos, políticos e acadêmicos contribuíram para a formação da pósgraduação no Brasil. Merece destaque a criação, em 1951, de agências de fomento ao desenvolvimento científico, entre elas a Coordenação de Aperfeiçoamento de Pessoal de Nível Superior (Capes), voltada à formação do magistério de nível superior.

Essas transformações foram acompanhadas de progressiva preocupação dos órgãos governamentais com a implementação de mecanismos voltados para a avaliação do desempenho desses cursos, visando à expansão e à consolidação da pós-graduação no país.

Segundo Lapa e Neiva (1996), as atividades de avaliação do desempenho são essenciais à legitimação social das organizações de ensino, uma vez que as instituições universitárias são dependentes de contínua legitimação pública, pois tanto os agentes internos quanto a sociedade em geral possuem expectativas em relação ao desempenho e à contribuição social dessas organizações.

Os autores destacam ainda que, no Brasil, apesar de as expectativas sociais de maior desempenho recairem, tradicionalmente, sobre as instituições criadas pelo Poder Público, elas também atingem as instituições privadas de ensino, na medida em que os resultados alcançados pelas primeiras tornam-se padrões de desempenho para toda a sociedade. Dessa forma, a necessidade de legitimação é comum às instituições públicas e privadas.

Além disso, a avaliação de desempenho aponta como importante instrumento de gestão das instituições de ensino, visto que os melhores resultados observados podem servir de modelo para as demais unidades analisadas.

Contudo, segundo Belloni, Magalhães e Sousa (2003), ainda percebe-se grande amadorismo nas práticas de avaliação institucional e de políticas públicas em educação, sendo necessário maior aprofundamento teórico e metodológico nessa área.

Mello et al. (2001) destacam que os procedimentos em uso são baseados em conceitos essencialmente qualitativos e fortemente dependentes da opinião do avaliador. Quando utilizadas versões quantitativas, ocorre a aplicação de índices brutos que não fornecem subsídios necessários a uma análise mais acurada.

A preocupação com o desempenho, conforme afirma Belloni (2000), não tem sido traduzida na construção de modelos de avaliação que explicitem o significado das variáveis e dos indicadores relativos a esse desempenho. Salienta ainda que, para a construção de uma metodologia de avaliação do desempenho, é necessário explicitar os critérios e construir indicadores das unidades educacionais em análise, os quais tenham clareza conceitual, viabilidade operacional e que sejam globais, contemplando simultaneamente os diversos fatores determinantes do trabalho acadêmico e suas inter-relações.

No caso específico dos programas de pós-graduação, a Capes avalia o desempenho dos cursos desde 1976 e, devido à sua experiência acumulada, com um programa de avaliação indiscutivelmente pioneiro, o Brasil tornou-se o país que mais contribuiu com o desenvolvimento da história organizada da avaliação institucional na América Latina (LeITE, 2002). Essa experiência avaliativa consolidou-se ao longo dos anos, sempre em diálogo com as instituições de ensino superior (IES), sendo considerada grande avanço em sistemas de avaliação educacional. 
O sistema de avaliação dos programas de pós-graduação da Capes inclui critérios qualitativos e quantitativos, contudo ambos são convertidos em considerações qualitativas e, ao final da avaliação, com base nas apreciações realizadas, estabelece-se um conceito numérico que varia de 1 a 7 , dimensionando a qualidade dos programas avaliados.

Destacam-se, entre os itens avaliados pela Capes, as considerações sobre as condições de oferta dos cursos (ex: qualificação do corpo docente e adequação do currículo), bem como a análise dos resultados provenientes das atividades dos programas de pós-graduação (produção científica e formação de pessoal qualificado).

Dessa forma, presumindo que as condições de oferta dos cursos estejam refletidas no resultado alcançado pelo programa, neste estudo serão utilizados como critérios para a análise do desempenho apenas os resultados derivados das atividades dos programas de pósgraduação.

O presente estudo, então, visa desenvolver uma análise do desempenho com referência à eficiência relativa de programas de pós-graduação, no intuito de conhecer o perfil do desempenho desses programas. Sua contribuição baseia-se na aplicação de um método para avaliação isento da opinião de avaliadores e que, ao mesmo tempo, busca respeitar as especificidades das unidades analisadas.

Ressalta-se não ser o objetivo desta pesquisa desenvolver modelo que venha substituir a metodologia atual, uma vez que a natureza dos programas de pós-graduação exige a utilização de modelos complexos para sua avaliação, envolvendo considerações minuciosas, de forma a contemplar todos os fatores que demonstrem o nível de qualidade dos cursos. Todavia, pretende-se apresentar modelo que possibilite reflexões sobre alternativas de melhoria do desempenho desses programas.

O trabalho apresenta-se em quatro capítulos, nos quais serão discutidos, nesta ordem, alguns conceitos associados à avaliação de instituições de ensino; os procedimentos metodológicos adotados; os resultados alcançados; e as considerações finais do estudo.

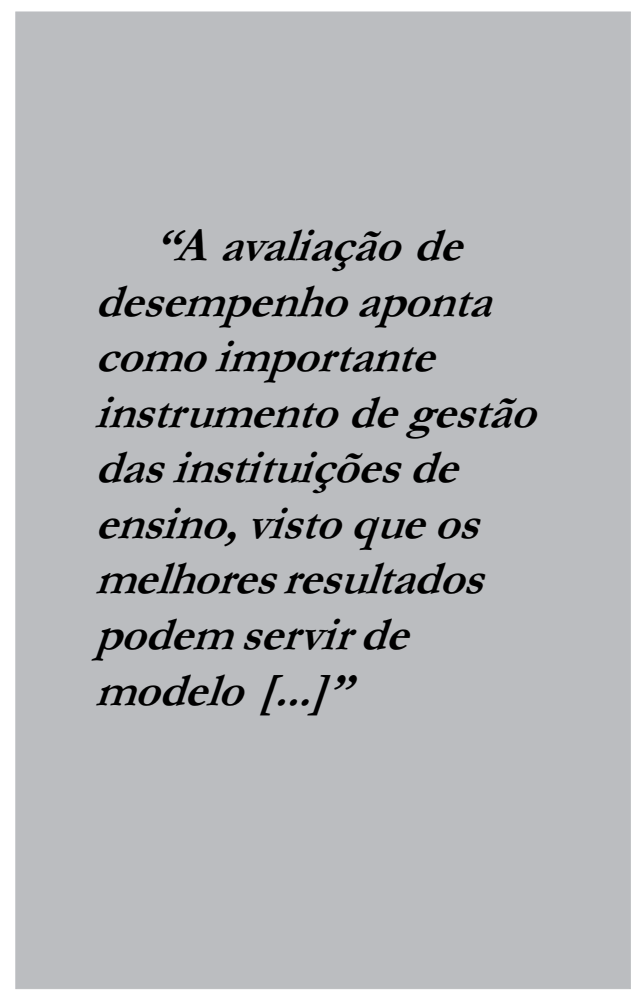

\section{Avaliação de desempenho em instituições de ensino}

Lapa e Neiva (1996) classificam os critérios de avaliação institucional em dois grandes grupos: os ligados à ideia de desempenho, compreendendo a avaliação da produtividade, eficiência, eficácia e 
efetividade; e aqueles voltados à ideia de qualidade, nos quais a avaliação ocorre a partir do ponto de vista da utilidade e relevância, conceitos relacionados às visões "políticas" de valor e que dependem da visão dos avaliadores.

Belloni (2000) define a ideia de avaliação de instituições de ensino voltada ao desempenho organizacional e, dessa perspectiva, decorrem três dimensões distintas de avaliação: 1) dimensão técnicooperacional, cujos critérios de avaliação são a produtividade e a eficiência, dados os recursos e conhecidos os produtos; 2) dimensão pedagógica, relacionada aos processos educacionais propriamente ditos, cujo critério de avaliação é a eficácia; e 3) dimensão política, que avalia a missão institucional, cujo critério de avaliação é a efetividade das ações da instituição.

Em suma, os critérios de avaliação institucional comumente encontrados na literatura podem ser classificados em dois grandes grupos: os relacionados ao desempenho organizacional e os que tratam da qualidade institucional. De acordo com os objetivos deste trabalho, maior esforço será despendido à definição de eficiência, conceito relacionado ao desempenho organizacional.

Lapa e Neiva (1996) destacam que a eficiência está ligada à ideia de possibilidades de trabalho em termos de geração de produtos e de configuração de resultados alcançáveis com os recursos disponíveis e os insumos utilizados, comparativamente a uma fronteira de desempenho possível de ser alcançada. Salientam ainda que o conceito de eficiência das instituições de ensino deve ser visto sob três perspectivas:

Eficiência técnica: avalia-se a possibilidade de aumentar a produção, mantendo-se a combinação de recursos ou a forma de trabalho, ou seja, sem alterar o projeto acadêmico e pedagógico da instituição.
Eficiência alocativa: verifica-se a possibilidade de aumentar a geração de algum produto, ou reduzir o consumo de algum insumo, mantendo-se a produção ou o consumo dos demais itens no nível atual, alterando apenas os processos de produção, ou seja, modificando os projetos acadêmicos e pedagógicos que orientam a instituição.

Eficiência tecnológica: avalia-se a possibilidade de melhorar quantitativamente ou qualitativamente os produtos e resultados gerados, agindo sobre a composição dos recursos, dos insumos e da tecnologia utilizada.

Comparando esses termos com aqueles utilizados nas organizações empresariais, nota-se uma pequena desconexão. No ramo empresarial, a eficiência técnica é avaliada a partir da melhor composição dos insumos para geração do volume máximo de produtos. Em instituições de ensino, no entanto, percebe-se que a eficiência técnica deve ser vista como a busca por melhores resultados, sem alterações na composição dos insumos.

$\mathrm{Na}$ administração de organizações lucrativas, a eficiência alocativa engloba questões acerca do preço dos insumos e produtos gerados pelo sistema avaliado. Já nas instituições educacionais, a eficiência alocativa é definida como a procura pela melhor composição dos recursos para gerar mais produtos, ou consumir menos insumos, não fazendo referência ao preço dos insumos e produtos.

O conceito de eficiência tecnológica das organizações de ensino faz analogia ao conceito de eficiência econômica das organizações lucrativas, em que é analisado o efeito conjunto da eficiência técnica e alocativa. Contudo, a eficiência tecnológica das instituições de ensino visa ao ganho na qualidade e na quantidade, a partir de alterações tanto no nível de insumos e produtos quanto na tecnologia empregada, 
não fazendo nenhuma menção ao valor econômico dos produtos.

Verifica-se que a caracterização de eficiência no setor público difere da utilizada em organizações produtivas, nas quais os custos dos insumos e o preço dos produtos são conhecidos e a tecnologia utilizada é relativamente padronizada. Nesse sentido, Lapa e Neiva (1996) sugerem que o cálculo da efíciência de organizações de ensino deva ser feito em termos relativos, tomando-se como referência uma ou algumas instituições que, em dado contexto similar ou equivalente, possam ser consideradas mais eficientes.

Vale destacar que o termo eficiência utilizado neste estudo refere-se, conjuntamente, às eficiências técnica, alocativa e tecnológica. Isso se dá pela impossibilidade, dada a técnica utilizada no estudo, de diferenciar os efeitos da alteração na composição dos insumos e nos projetos pedagógicos dos programas de pós-graduação, o que demandou técnica de análise relativa da eficiência que considere as especificidades das instituições de ensino superior.

\section{Procedimentos metodológicos}

\section{Unidades de análise}

Considerando o expressivo crescimento do número de programas de pós-graduação acadêmicos em Administração, Contabilidade e Turismo (Tabela 1), bem como a carência de estudos que utilizem métodos quantitativos para a avaliação nessa área, os referidos programas serão as unidades analisadas nesta pesquisa.

Visando garantir uniformidade e contemporaneidade à análise, uma vez que os critérios utilizados na avaliação dos programas de pós-graduação são revistos e ajustados a cada triênio, optou-se por trabalhar com os programas de pósgraduação acadêmicos em Administração, Contabilidade e Turismo avaliados pela Capes no triênio 2004/2006.

Assim, a amostra da pesquisa é representada, nos anos de 2004, 2005 e 2006, por um total de 44, 54 e 60 programas de pós-graduação, respectivamente. Entretanto, ressalta-se que foram eliminados do estudo, em 2004, dois programas de pósgraduação; cinco programas em 2005; e quatro em 2006. A exclusão de um deles se deu em razão do não fornecimento de dados necessários à análise, apesar das insistentes tentativas junto à instituição de ensino do referido programa. Os demais foram eliminados porque já estavam em funcionamento antes de serem credenciados pelo órgão regulador. Essa exclusão é justificada pela impossibilidade de se distinguir os resultados decorrentes do trabalho acadêmico antes e após a autorização oficial.

Dessa forma, o estudo contemplou, nos anos de 2004, 2005 e 2006, respectivamente, 42, 49 e 56 programas

Tabela 1: Evolução do número de programas de pós-graduação acadêmicos em Administração, Contabilidade e Turismo, avaliados pela Capes - Brasil, 1996-2006

\begin{tabular}{cccccccccccc}
\hline & 1996 & 1997 & 1998 & 1999 & 2000 & 2001 & 2002 & 2003 & 2004 & 2005 & 2006 \\
\hline Programas & 23 & 22 & 19 & 25 & 28 & 31 & 34 & 40 & 44 & 54 & 60 \\
\hline
\end{tabular}

Fonte: Adaptado da Capes (2007c). 
de pós-graduação acadêmicos em Administração, Contabilidade e Turismo.

\section{Referencial analítico}

Na construção metodológica, utilizouse a Análise Envoltória de Dados (DEA), um modelo não paramétrico, para mensurar a eficiência dos programas de pós-graduação acadêmicos em Administração, Contabilidade e Turismo.

Alguns autores, como Paiva (2000), Mello et al. (2003), Angulo-Meza et al. (2003) e Estellita Lins, Almeida e Bartholo Junior (2004), também utilizaram a DEA para analisar o desempenho de programas de pós-graduação no Brasil, avaliando programas da área de Engenharia.

Em termos matemáticos, a DEA calcula a eficiência por meio da razão entre a soma ponderada de saídas (outputs) e a soma ponderada de entradas (inputs). O peso para cada fator de ponderação (insumos e produtos) é obtido a partir da resolução de problema de programação fracionária, em que cada unidade analisada maximiza sua eficiência (MELlo et al., 2003).

$\mathrm{Na}$ versão inicial da DEA, as unidades analisadas eram supostamente capazes de tomar decisões sobre o nível de insumos utilizados e sobre a quantidade de produtos resultantes, sendo consideradas unidades tomadoras de decisão ou DMU - Decision Making Units (MELLo et al., 2001).

A DEA apresenta dois modelos básicos de análise. Há o "modelo com retornos constantes", ou "modelo CCR", desenvolvido por Charnes, Cooper e Rhodes; e o "modelo BCC", também conhecido por "modelo com retornos variáveis", desenvolvido por Banker, Charnes e Cooper (Gomes e BApTisTa, 2004).

No presente trabalho, optou-se pelo modelo com retornos constantes à escala (CCR), por não se presumir a existência de efeitos de escala nas atividades desenvolvidas pelos programas de pós-graduação. Essa escolha é condizente com o tratamento analítico adotado nos estudos de Mello et al. (2003), Angulo-Meza et al. (2003) e Estellita Lins, Almeida e Bartholo Junior (2004).

Tanto o modelo CCR quanto o BCC podem ser analisados pela ótica da orientação para insumos ou pela orientação para o produto. Segundo Gomes e Baptista (2004), a escolha da orientação não exerce grande influência na magnitude do valor de eficiência técnica, e o critério de escolha dependerá da finalidade do estudo, ou seja, se o objetivo é regularizar o consumo de insumos ou possibilitar o aumento da produção.

Optou-se também por trabalhar com o modelo de análise de eficiência orientado para o produto, uma vez que a busca da eficiência por meio da maximização da produção apresenta-se mais condizente à natureza das atividades dos programas de pós-graduação.

Para Estellita Lins e Angulo-Meza (2000), o método DEA com orientaçãoproduto e que pressupõe retornos constantes de escala (CCR) é modelado em (1), em que $y_{k}$ é um vetor $(m \times 1)$ de quantidades de produto da k-ésima DMU; $x_{k}$ é um vetor $(s \times 1)$ de quantidade de insumos da k-ésima DMU; $Y$ é uma matriz (n $\mathrm{x} \mathrm{m}$ ) de produtos das $n$ DMUs; $X$ é uma matriz $(n \times s)$ de insumos das $n$ DMUs; $\lambda$ é um vetor $(n \times 1)$ de pesos; e $\varnothing$ é uma escalar que tem valores iguais ou maiores do que 1 e indica o escore de eficiência das DMUs, em que valor igual a 1 indica a eficiência técnica relativa da k-ésima DMU em relação às demais, e valor superior a 1 indica ineficiência técnica relativa. O valor obtido subtraindo-se $\varnothing$ de 1 indica o aumento pro- 
porcional nos produtos que a k-ésima DMU pode alcançar, mantendo-se constante a quantidade de insumos.

$$
\begin{aligned}
& \max _{\phi}, \lambda \phi, \\
& \text { sujeito a : } \\
& \phi y_{k}-Y \lambda \leq 0, \\
& -x_{k}+X \lambda \leq 0, \\
& -\lambda \leq 0
\end{aligned}
$$

Esse modelo busca maximizar a produção, utilizando no máximo o volume de insumos observados, e pressupõe retornos constantes de escala. Assim, para $n$ DMUs, considerando-se $m$ inputs e $s$ outputs, a eficiência é obtida quando se alcança a máxima produção, dados os insumos produtivos consumidos. O escore de efíciência adotado no presente estudo será obtido pelo inverso da medida escalar; portanto, o escore de eficiência gerado está compreendido entre 0 e 1 .

Ressalta-se que, por ser a DEA modelo mais objetivo na avaliação da eficiência de unidades produtivas, uma vez que elimina a subjetividade no estabelecimento de pesos aos fatores (insumos e produtos), ela permite que esse peso seja determinado da maneira mais favorável a cada unidade analisada (MeLLo et al., 2001). Contudo, quando o modelo é composto por grande número de variáveis, uma DMU pode ser considerada eficiente ao serem atribuídos pesos nulos a quase todos os coeficientes, de tal forma que vários fatores deixam de ser considerados na avaliação, tornando-a incompleta.

Acreditando ser importante a utilização de um conjunto maior de variáveis, tendo em vista a natureza complexa das unidades analisadas, buscou-se contemplar neste estudo aqueles fatores essenciais ao desenvolvimento das atividades dos programas de pós-graduação. Para maximizar o poder discricionário do modelo utilizado, optou-se por agrupar certos fatores com sentido semelhante, de forma a reduzir o número de variáveis empregadas na mensuração da eficiência relativa e evitar que algumas delas deixassem de ser contempladas na avaliação.

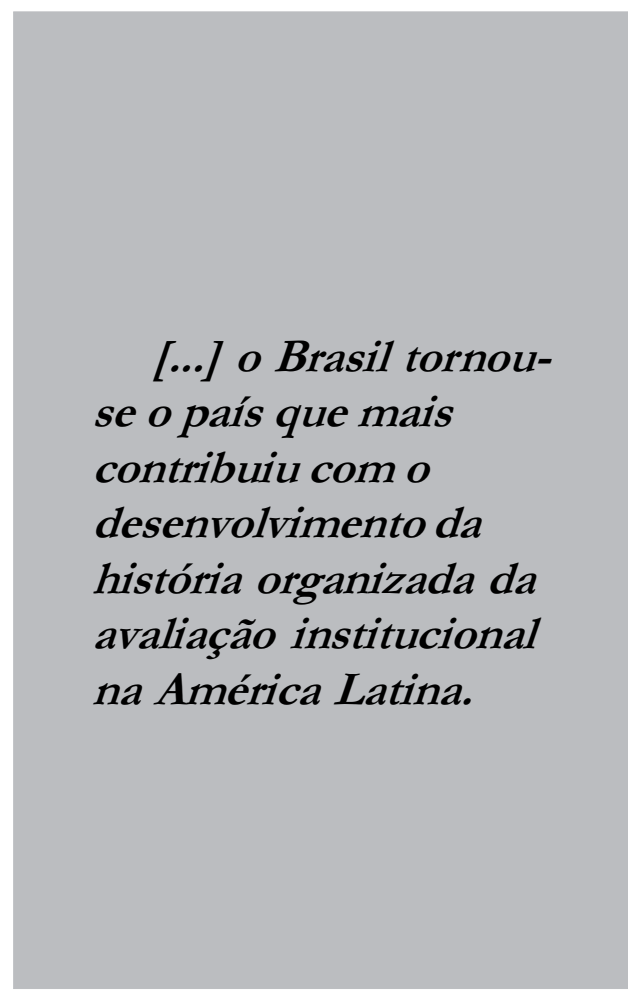

Variáveis de insumos (x)

Conhecidas as defasagens entre o ingresso dos alunos nos cursos de mestrado e doutorado e as suas respectivas titulações - cerca de dois anos no mestrado e quatro anos no doutorado (Tabela 2) -, foi necessário tratar a variável relacionada aos alunos de forma distinta nesses dois níveis de cursos, pois alguns programas de pós-graduação oferecem apenas o curso 
Tabela 2: Tempo médio para titulação dos alunos dos cursos de pós-graduação acadêmicos em Administração, Contabilidade e Turismo - Brasil, 2004-2006

\begin{tabular}{lllll}
\hline \multirow{2}{*}{ Anos } & \multicolumn{3}{c}{ Mestrado } & \multicolumn{2}{c}{ Doutorado } \\
\cline { 2 - 5 } & Meses & Anos & Meses & Anos \\
\hline 2004 & 29,4 & 2,5 & 55,7 & 4,6 \\
\hline 2005 & 29,7 & 2,5 & 51,2 & 4,3 \\
\hline 2006 & 28,2 & 2,3 & 47,6 & 4,0 \\
\hline
\end{tabular}

Fonte: Capes (2007b).

de mestrado, enquanto outros também oferecem o curso de doutorado.

Dessa forma, empregou-se no modelo de análise o seguinte conjunto de variáveis:

DOCPER - número de docentes permanentes do programa.

INGTOT - número total de alunos ingressantes no programa de pósgraduação, representado pela soma do número de alunos que ingressaram nos cursos de mestrado e doutorado, respectivamente, dois e quatro anos antes do período em análise.

\section{Variáveis de produtos (y)}

Observa-se uma série de variáveis que se apresentam como resultantes das atividades dos programas de pós-graduação. Assim, buscou-se incluir no modelo os principais fatores relacionados à produção dos programas, como a formação de recursos humanos, ou seja, a titulação de mestres e doutores, e a publicação científica.

Com relação à publicação científica, foi necessária a construção de um indicador para compor essa variável, dada a multiplicidade de tipos e veículos. Para isso, utilizou-se a soma ponderada das publicações do programa, a partir dos pesos aplicados na avaliação realizada pela Capes no triênio 2004/2006 (CAPES, 2007a). Esse indicador é composto pelos artigos publicados em periódicos de circulação internacional, nacional e local; artigos completos publicados em anais de congressos; livros e capítulos de livros publicados.

Dessa forma, empregou-se o seguinte conjunto de variáveis de produtos:

TITTOT - número total de alunos de mestrado e doutorado titulados no programa de pós-graduação, no ano em análise.

PUBCIE - publicação científica do programa de pós-graduação, representada pela soma ponderada das produções científicas vinculadas ao programa, com autoria ou coautoria de docentes permanentes.

\section{Fonte de dados}

Para a realização da pesquisa, foi utilizado o banco de dados disponibilizado pela Capes na internet, com os indicadores resultantes da referida avaliação (CAPES, 2007a, 2007b, 2007c).

\section{Resultados e discussão}

$\mathrm{Na}$ Tabela 3, são apresentadas as variáveis consideradas na mensuração da eficiência relativa dos programas de pósgraduação, bem como suas estatísticas descritivas, no período de 2004 a 2006. 
Percebe-se, por meio das variáveis DOCPER e INGTOT, uma relativa diferença de magnitudes entre os programas avaliados. Verifica-se que alguns programas contam, nos três anos analisados, com um corpo docente de 48 professores e cerca de 80 alunos ingressantes em cursos de mestrado e doutorado, enquanto outros possuem entre três e oito professores em seu corpo docente e até mesmo nenhum aluno ingressante em cursos de mestrado e doutorado.

É oportuno salientar que, no período de 2004 a 2006, os programas de pósgraduação apresentaram médias decrescentes em relação à composição do corpo docente permanente e ao número de alunos que ingressaram e de alunos titulados. Isso pode ser decorrente da criação de novos cursos, uma vez que os programas novos iniciam suas atividades operando em menor proporção, se comparados aos programas estabelecidos há mais tempo.

No intuito de verificar o impacto da criação dos cursos nessas variáveis, foram calculadas as estatísticas descritivas, considerando apenas os 42 programas em funcionamento desde o início do triênio (Tabela 4).

Portanto, considerando apenas os programas em funcionamento desde 2004, nota-se que a composição do corpo docente permanente, o número de alunos que ingressaram e de alunos titulados apresentaram médias crescentes ao longo do triênio analisado, comprovando a

Tabela 3: Estatística descritiva das variáveis empregadas na mensuração da eficiência dos programas de pós-graduação acadêmicos em Administração, Contabilidade e Turismo - Brasil, 2004-2006

\begin{tabular}{llcccc}
\hline Ano & Estatísticas & DOCPER & INGTOT & TITTOT & PUBCIE \\
\hline \multirow{2}{*}{2004} & Número de programas & 42 & 42 & 42 & 42 \\
Média & 14,9 & 27,4 & 23,6 & 392,32 \\
& Desvio-padrão & 8,2 & 20,9 & 19,8 & 343,92 \\
& Máximo & 48 & 96 & 108 & 1978,79 \\
& 7 & 0 & 0 & 88,08 \\
Núnimo & 49 & 49 & 49 & 49 \\
Mémero de programas & 14,1 & 23,4 & 21,6 & 417,90 \\
Desvio-padrão & 7,4 & 19,9 & 18,5 & 393,54 \\
& Máximo & 48 & 81 & 103 & 2413,72 \\
& Mínimo & 8 & 0 & 0 & 78,68 \\
& Número de programas & 56 & 56 & 56 & 56 \\
Média & 13,9 & 22,2 & 20,4 & 293,76 \\
Desvio-padrão & 7,7 & 18,5 & 16,2 & 264,45 \\
Máximo & 48 & 80 & 66 & 1599,99 \\
Mínimo & 3 & 0 & 0 & 22,64 \\
\hline
\end{tabular}

Fonte: Resultados da pesquisa. 
Tabela 4: Estatística descritiva das variáveis empregadas na mensuração da eficiência dos programas de pós-graduação acadêmicos em Administração, Contabilidade e Turismo, considerando apenas os programas em funcionamento desde o início do triênio - Brasil, 2004-2006

\begin{tabular}{llrrr}
\hline Ano & Estatísticas & DOCPER & INGTOT & TITTOT \\
\hline \multirow{2}{*}{2004} & Número de programas & 42 & 42 & 42 \\
& Média & 14,9 & 27,4 & 23,6 \\
& Desvio-padrão & 8,2 & 20,9 & 19,8 \\
& Máximo & 48 & 96 & 108 \\
& Mínimo & 7 & 0 & 0 \\
& Número de programas & 42 & 42 & 42 \\
& Média & 14,9 & 27,4 & 25,2 \\
& Desvio-padrão & 7,7 & 18,9 & 17,6 \\
& Máximo & 48 & 81 & 103 \\
& Mínimo & 8 & 0 & 0 \\
& Número de programas & 42 & 42 & 42 \\
& Média & 15,4 & 29,0 & 26,5 \\
& Desvio-padrão & 8,3 & 16,0 & 14,0 \\
& Máximo & 48 & 80 & 66 \\
& Mínimo & 7 & 4 & 4 \\
\hline
\end{tabular}

Fonte: Resultados da pesquisa.

influência dos novos cursos na magnitude dos programas.

Com relação à variável PUBCIE, percebe-se aumento da produção científica do ano de 2004 para 2005, e uma redução no ano de 2006. Contudo, não se pode afirmar que houve queda na produtividade dos programas avaliados no ano de 2006, pois os pesos utilizados no cômputo dessa variável diferem dos empregados em 2004 e 2005, conforme os critérios utilizados pela Comissão de Avaliação da área Administração/Turismo.

Nota-se ainda elevado desvio-padrão, decorrente da grande dispersão dos dados em torno da média. Esse alto desviopadrão compromete a utilização de técnicas estatísticas paramétricas, uma vez que não se pode assumir nenhuma forma de distribuição dos dados, prejudicando a utilização de medidas de tendência central.

Essa limitação dos dados é suprida pela utilização da Análise Envoltória de Dados (DEA), técnica não paramétrica que não pressupõe normalidade ou qualquer forma de distribuição dos dados.

A Tabela 5 mostra as estatísticas descritivas dos escores de eficiência dos programas de pós-graduação acadêmicos em Administração, Contabilidade e Turismo, gerados por meio da DEA, com orientação para o produto e retornos constantes à escala.

Verifica-se que os programas de pós-graduação apresentaram-se, em média, mais eficientes no ano de 2006, seguidos 
Tabela 5: Estatística descritiva dos escores de eficiência gerados para os programas de pós-graduação acadêmicos em Administração, Contabilidade e Turismo-Brasil, 2004-2006

\begin{tabular}{lrrr}
\hline Estatísticas & $\mathbf{2 0 0 4}$ & $\mathbf{2 0 0 5}$ & $\mathbf{2 0 0 6}$ \\
\hline Programas avaliados & 42 & 49 & 56 \\
Média & 0,6979 & 0,6457 & 0,7493 \\
Desvio-padrão & 0,1940 & 0,2232 & 0,2082 \\
Coeficiente de variação & 0,2780 & 0,3458 & 0,2779 \\
Máximo & 1,0000 & 1,0000 & 1,0000 \\
Mínimo & 0,1640 & 0,1547 & 0,0780 \\
Amplitude & 0,8360 & 0,8453 & 0,9220 \\
Assimetria & $-0,5571$ & $-0,1123$ & $-1,2005$ \\
\hline
\end{tabular}

Fonte: Resultados da pesquisa.

pelos escores de eficiência médios dos anos de 2004 e 2005, respectivamente.

Considerando que o escore médio de eficiência em 2004 foi de 69,79\% (Tabela 5), pode-se constatar que os programas de pós-graduação analisados poderiam aumentar, em média, 30,21\% do nível de produção nesse período. Esse percentual passa para 35,43\% em 2005 e 25,07\% em 2006. Isso significa que os programas de pós-graduação atingiram, em média, resultados abaixo do potencial no triênio 2004/2006, se comparados aos melhores desempenhos observados em cada ano.

Nota-se que, em relação à distribuição dos escores de eficiência, o ano de 2005 apresentou maior dispersão em torno da média. Contudo, no ano de 2006 pôde-se observar maior amplitude entre os valores dos escores de eficiência, ou seja, apesar de os programas alcançarem, em média, maior eficiência nesse ano, o menor escore de eficiência do triênio refere-se ao ano de 2006.

É possível constatar discreta assimetria em 2004 e 2005. Já em 2006, notou-se significativa assimetria, demonstrando que os valores encontravam-se mais concentrados à direita, ou seja, nos maiores.

$\mathrm{Na}$ Tabela 6, é apresentada a distribuição de frequência dos escores de eficiência técnica, gerados para os programas de pós-graduação acadêmicos em Administração, Contabilidade e Turismo.

No ano de 2004, foram avaliados 42 programas de pós-graduação e a maior concentração dos índices de eficiência refere-se ao intervalo dos escores entre $70 \%$ e $80 \%$, no qual se encontram 12 programas $(28,6 \%)$. Vale ressaltar que o segundo maior percentual de programas de pós-graduação com eficiência igual ou superior a 90\% encontra-se no ano de 2004, no qual oito programas $(19 \%)$ alcançaram esse resultado.

Por sua vez, em 2005, foram analisados 49 programas, e os escores de eficiência apresentam-se mais concentrados entre $40 \%$ e $50 \%$, englobando 10 programas de pós-graduação $(20,4 \%)$. Destaca-se que o menor percentual de programas com escores de eficiência iguais 
Tabela 6 - Distribuição dos programas de pós-graduação acadêmicos em Administração, Contabilidade e Turismo, por nível de eficiência - Brasil, 2004-2006

\begin{tabular}{lrrrrrrrrr}
\hline Intervalo de & \multicolumn{3}{c}{ Número de Ocorrência } & \multicolumn{3}{c}{ Frequência Relativa( $\%)$} & \multicolumn{4}{c}{ Frequência Acumulada (\%) } \\
\cline { 2 - 10 } Eficiência & 2004 & 2005 & 2006 & 2004 & 2005 & 2006 & 2004 & 2005 & 2006 \\
\hline $90 \%-100 \%$ & 8 & 7 & 14 & $19,0 \%$ & $14,3 \%$ & $25,0 \%$ & $19,0 \%$ & $14,3 \%$ & $25,0 \%$ \\
$80 \%-90 \%$ & 1 & 6 & 12 & $2,4 \%$ & $12,2 \%$ & $21,4 \%$ & $21,4 \%$ & $26,5 \%$ & $46,4 \%$ \\
$70 \%-80 \%$ & 12 & 7 & 10 & $28,6 \%$ & $14,3 \%$ & $17,9 \%$ & $50,0 \%$ & $40,8 \%$ & $64,3 \%$ \\
$60 \%-70 \%$ & 9 & 9 & 9 & $21,4 \%$ & $18,4 \%$ & $16,1 \%$ & $71,4 \%$ & $59,2 \%$ & $80,4 \%$ \\
$50 \%-60 \%$ & 8 & 5 & 5 & $19,0 \%$ & $10,2 \%$ & $8,9 \%$ & $90,5 \%$ & $69,4 \%$ & $89,3 \%$ \\
$40 \%-50 \%$ & 1 & 10 & 2 & $2,4 \%$ & $20,4 \%$ & $3,6 \%$ & $92,9 \%$ & $89,8 \%$ & $92,9 \%$ \\
$30 \%-40 \%$ & 0 & 1 & 1 & $0,0 \%$ & $2,0 \%$ & $1,8 \%$ & $92,9 \%$ & $91,8 \%$ & $94,6 \%$ \\
$20 \%-30 \%$ & 2 & 3 & 2 & $4,8 \%$ & $6,1 \%$ & $3,6 \%$ & $97,6 \%$ & $98,0 \%$ & $98,2 \%$ \\
$10 \%-20 \%$ & 1 & 1 & 0 & $2,4 \%$ & $2,0 \%$ & $0,0 \%$ & $100,0 \%$ & $100,0 \%$ & $98,2 \%$ \\
$0 \%-10 \%$ & 0 & 0 & 1 & $0,0 \%$ & $0,0 \%$ & $1,8 \%$ & $100,0 \%$ & $100,0 \%$ & $100,0 \%$ \\
\hline
\end{tabular}

Fonte: Resultados da pesquisa.

ou superiores a $90 \%$ refere-se ao ano de 2005, em que apenas $14,3 \%$ dos programas analisados alcançaram tal resultado.

Já no ano de 2006, encontra-se o maior percentual de programas de pósgraduação com eficiência igual ou superior a $90 \%$, sendo 14 programas avaliados (25\%). A maior concentração dos índices de eficiência em 2006 também refere-se ao intervalo de $90 \%$ a $100 \%$.

Também se buscou conhecer a distribuição da eficiência dos programas de pós-graduação, segundo a dependência administrativa das instituições às quais os programas são vinculados, ou seja, verificar a diferença entre o desempenho médio alcançado pelos programas de pós-graduação vinculados à administração pública e o desempenho dos programas das instituições privadas.

Dessa forma, a Tabela 7 mostra os escores médios de eficiência relativa dos programas de pós-graduação acadêmicos em Administração, Contabilidade e Turismo, segundo a dependência administrativa.

Observa-se que os programas de pósgraduação das instituições privadas de ensino apresentam-se, em média, mais eficientes do que os programas da rede pública. Além disso, pode-se notar maior dispersão nos escores dos programas vinculados às instituições públicas de ensino, denotando maior disparidade no nível de eficiência desses programas.

Com a análise da eficiência relativa dos programas de pós-graduação, os programas considerados ineficientes podem adotar a estratégia de benchmarking, no sentido de incorporar ações qualitativas observadas nos programas tidos como eficientes.

Percebe-se que, com exceção de 2005, a maior amplitude dos escores de eficiência encontra-se nos programas de pósgraduação das instituições públicas. 
Tabela 7: Estatística descritiva dos escores de eficiência dos programas de pós-graduação acadêmicos em Administração, Contabilidade e Turismo, por dependência administrativa - Brasil, 2004-2006

\begin{tabular}{|c|c|c|c|c|c|c|c|c|}
\hline \multirow{2}{*}{ Ano } & \multirow{2}{*}{$\begin{array}{l}\text { Dependência } \\
\text { administrativa }\end{array}$} & \multicolumn{2}{|c|}{ Programas } & \multirow{2}{*}{ Média } & \multirow{2}{*}{$\begin{array}{l}\text { Desvio- } \\
\text { padrão }\end{array}$} & \multirow{2}{*}{$\begin{array}{c}\text { Coeficiente } \\
\text { de } \\
\text { variação }\end{array}$} & \multirow{2}{*}{ Amplitude } & \multirow{2}{*}{ Assimetria } \\
\hline & & Nr. & $\%$ & & & & & \\
\hline \multirow{2}{*}{2004} & Privada & 18 & 42,86 & 0,7362 & 0,1763 & 0,2394 & 0,7144 & $-0,7004$ \\
\hline & Pública & 24 & 57,14 & 0,6691 & 0,2052 & 0,3067 & 0,8360 & $-0,4310$ \\
\hline \multirow{2}{*}{2005} & Privada & 22 & 44,90 & 0,6601 & 0,2191 & 0,3319 & 0,8453 & $-0,3475$ \\
\hline & Pública & 27 & 55,10 & 0,6339 & 0,2300 & 0,3629 & 0,7733 & 0,0585 \\
\hline \multirow{2}{*}{2006} & Privada & 26 & 46,43 & 0,7569 & 0,2020 & 0,2668 & 0,7904 & $-1,0044$ \\
\hline & Pública & 30 & 53,57 & 0,7427 & 0,2168 & 0,2919 & 0,9220 & $-1,3798$ \\
\hline
\end{tabular}

Fonte: Resultados da pesquisa.

É possível constatar discreta assimetria nos níveis de eficiência em 2004 e 2005. Observa-se, ainda, que em 2006 existia uma significativa assimetria, indicando que os escores de eficiência encontravam-se concentrados nos maiores valores, tanto nas instituições privadas de ensino quanto nas públicas.

Os fatores que levam à diferença no nível de eficiência entre programas de pósgraduação de instituições privadas e públicas de ensino podem estar associados a processos pedagógicos, ou até mesmo gerenciais.

Destaca-se que os docentes da rede pública de ensino, além das atividades da pós-graduação, atuam também no ensino de graduação e em trabalhos de extensão e, frequentemente, estão vinculados a cargos administrativos na própria instituição, de forma que o tempo dedicado às atividades de pesquisa e pós-graduação pode estar sendo comprometido, prejudicando a eficiência dos programas.

Contudo, para uma análise mais conclusiva, faz-se necessário maior aprofundamento sobre os fatores associados à eficiência desses programas.

\section{Considerações finais}

Após análise dos programas de pósgraduação acadêmicos em Administração, Contabilidade e Turismo, pôde-se verificar significativa diferença de magnitude entre eles, decorrente, em tese, do expressivo aumento do número de cursos nessa área do conhecimento, uma vez que os cursos novos iniciam suas atividades em menor escala.

Os resultados do modelo de mensuração da eficiência proposto apontaram, no triênio em estudo, maior nível de eficiência dos programas de pós-graduação no ano de 2006, seguido pelos anos de 2004 e 2005, respectivamente. Entretanto, se avaliado o desempenho médio dos programas de pósgraduação, constata-se que vêm atingindo resultados abaixo do potencial, se comparados aos melhores resultados observados em cada ano.

Verifica-se, entre os programas de pósgraduação acadêmicos em Administração, Contabilidade e Turismo, expressiva participação de cursos da iniciativa privada. Analisando a eficiência por dependência administrativa, pôde-se observar que, em 
média, os programas vinculados às instituições privadas apresentaram-se mais eficientes do que os programas da rede pública de ensino.

A análise dos projetos acadêmicos, da estrutura organizacional e, principalmente, da gestão dos recursos utilizados pelos programas de pós-graduação identificados como eficientes torna-se importante mecanismo de benchmarking para os programas menos eficientes. Dessa forma, o gestor da instituição pública ou privada poderia, a partir das análises, adotar as melhores práticas observadas, visando à maximização da eficiência em sua gestão.

(Artigo recebido em abril de 2009. Versão final em dezembro de 2009.)

\section{Referências bibliográficas}

Angulo-Meza, Lidia et al. Avaliação do Ensino nos Cursos de Pós-Graduação em Engenharia: um enfoque quantitativo de avaliação em conjunto. Engevista, Niterói, v. 5, n. 9, p. 41-49, 2003. Disponível em: <http://www.uff.br/engevista/sumário9.htm>. Acesso em: 27 mar. 2007.

Belloni, Isaura; Magalhães, Heitor de; Sousa, Luzia Costa de. Metodologia de avaliação em políticas públicas. 3. ed. São Paulo: Cortez, 2003. 96 p.

BELLONI, José Ângelo. Uma metodologia de avaliação da eficiência produtiva de universidades federais brasileiras. 2000. 245 p. Tese (Doutorado em Engenharia de Produção) - Universidade Federal de Santa Catarina, Florianópolis. Disponível em: <http://teses.eps.ufsc.br/ defesa/pdf/1757.pdf>. Acesso em: 23 ago. 2006.

Coordenação de Aperfeiçoamento de Pessoal de Nível Superior - Capes. Avaliação - Critérios de Avaliação - Avaliação Trienal 2007 (triênio 2004 - 2006) - Administração, Ciências Contábeis e Turismo. Disponível em: <http://www.capes.gov.br/avaliacao/criterios/ avaliacao_trienal_2007.html>. Acesso em: 9 jul. 2007a.

. Avaliação-Cadernos de Indicadores-Administração, Ciências Contábeis e Turismo.

Disponível em: <http://servicos.capes.gov.br/cadernosavaliacao/jsp/FiltraArquivos. jsp?\&Area=27\&IES=Nenhuma. Acesso em: 19 out. 2007b.

. Sobre a CAPES - Estatísticas. Disponível em: <http://ged.capes.gov.br/ $\mathrm{AgDw} /$ silverstream/pages/frPesquisaColeta.html>. Acesso em: 23 ago. 2007c.

Esteldita Lins, Marcos Pereira; Almeida, Bernardo Faria de; Bartholo Junior, Roberto. Avaliação de desempenho na pós-graduação utilizando a Análise Envoltória de Dados: o caso da Engenharia de Produção. In: Revista Brasileira de Pós-Graduação, n.1, p. 41-56, jul. 2004. Disponível em: < http://www2.capes.gov.br/rbpg/portal/conteudo/ 41_56_avaliacao_de_desempenho_na_pos.pdf>. Acesso em: 7 fev. 2007.

Esteilita Lins, Marcos Pereira; Angulo-Meza, Lídia (ed). Análise envoltória de dados e perspectivas de integração no ambiente do apoio à decisão. Rio de Janeiro: COPPE/UFRJ, 2000. 232 p. Gomes, Adriano Provezano; Baptista, Antônio José Medina dos Santos. Análise Envoltória de Dados: conceitos e modelos básicos. In: SAnTos, Maurinho Luiz dos; 
Vieira, Wilson da Cruz (ed). Métodos Quantitativos em Economia. Viçosa MG: Editora UFV, 2004. 653 p., p. 121-160.

Lapa, Jair dos Santos; NeIva, Cláudio Cordeiro. Avaliação em Educação: comentários sobre desempenho e qualidade. Ensaio: avaliação e políticas públicas em educação. Rio de Janeiro, v. 4 , n. 12 , p. 213-236, 1996.

LeITE, Denise. Sistemas de Avaliação das Instituições de Ensino Superior no Brasil. In: SoAres, Maria Suzana Arrosa (coord). A educação superior no Brasil. Brasília: Coordenação de Aperfeiçoamento de Pessoal de Nível Superior, 2002. p. 87-106.

MACCARI, Emerson Antonio et al. Sistema de avaliação da pós-graduação da Capes: pesquisa-ação em um programa de pós-graduação em Administração. Revista Brasileira de Pós-Graduação, Brasília, v. 5, n. 9, p. 171-205, 2008. Disponível em: <http:// www2.capes.gov.br/rbpg/index.php/numeros-publicados/volume-5-no-9> Acesso em: 27 nov. 2009.

Martins, Carlos Benedito. A Formação do Sistema Nacional de Pós-Graduação. In: Soares, Maria Suzana Arrosa (coord). A educação superior no Brasil. Brasília: Coordenação de Aperfeiçoamento de Pessoal de Nível Superior, 2002. p. 70-87.

Mello, João Carlos et al. Avaliação Qualitativa e Quantitativa: uma metodologia de integração. Ensaio: avaliação e políticas públicas em educação. Rio de Janeiro, v. 9, n. 31, p. 237-251, 2001.

Meldo, João Carlos Correia Baptista Soares de et al. Uma análise da qualidade e da produtividade de programas de pós-graduação em Engenharia. Ensaio: avaliação e políticas públicas em educação. Rio de Janeiro, v. 11, n. 39, p. 167-179, 2003.

Oliven, Arabela Campos. Histórico da Educação Superior no Brasil. In: SoAREs, Maria Suzana Arrosa (coord). A educação superior no Brasil. Brasília: Coordenação de Aperfeiçoamento de Pessoal de Nível Superior, 2002. p. 31-42.

PaIva, Francisco Canindé de. Eficiência produtiva de programas de ensino de pós-graduação em engenharias: uma aplicação do método análise envoltória de dados - DEA. 2000. 79p. Dissertação (Mestrado em Engenharia) - Universidade Federal de Santa Catarina, Florianópolis. Disponível em: < http://teses.eps.ufsc.br/defesa/pdf/4418.pdf > . Acesso em: 31 ago. 2006.

Teixeira, Anísio. Ensino Superior no Brasil: análise e interpretação de sua evolução até 1969. Rio de Janeiro: Editora da Fundação Getúlio Vargas, 1989. 186 p. 


\section{Resumo-Resumen-Abstract}

\section{Análise da eficiência relativa dos programas de pós-graduação acadêmicos em Administração, Contabilidade e Turismo}

Ney Paulo Moreira, Nina Rosa da Silveira Cunba, Marco Aurélio Marques Ferreira e Suely de Fátima

Ramos Silveira

O objetivo deste artigo é apresentar um modelo alternativo de análise da eficiência dos programas de pós-graduação acadêmicos em Administração, Contabilidade e Turismo, vinculados às instituições de ensino superior públicas e privadas. O estudo tem como base teórica a eficiência e a otimização de recursos, tomando como referência a maximização do retorno, sujeito às limitações de recursos. Como modelo analítico foi utilizada a Análise Envoltória de Dados (DEA), enquanto técnica não paramétrica de análise da eficiência relativa. Os resultados apontaram que os programas de pós-graduação foram mais eficientes em 2006, seguido por 2004 e 2005, respectivamente. Notouse ainda que, em média, os programas vinculados às instituições privadas de ensino foram mais eficientes que os da rede pública no triênio 2004/2006. Os gestores desses programas podem utilizar a análise da eficiência relativa como estratégia de benchmarking, adotando as melhores práticas observadas nos programas eficientes, visando à maximização da eficiência em sua gestão.

Palavras-chave: desempenho, Análise Envoltória de Dados.

Análisis de la eficiencia relativa de los programas de postgrado académicos en Administración, Contabilidad y Turismo

Ney Paulo Moreira, Nina Rosa da Silveira Cunha, Marco Aurélio Marques Ferreira y Suely de Fátima

Ramos Silveira

Este estudio tiene como objetivo presentar un modelo alternativo de análisis de la eficiencia para los programas de postgrado académicos en Administración, Contabilidad y Turismo vinculados a las instituciones de enseñanza superior públicas y privadas. El estudio tiene como base teórica la eficiencia y la optimización de recursos, tomando como referencia la maximización de retorno, sujeto a las limitaciones de recursos. Como modelo analítico fue utilizado Análisis Envoltorio de Datos (DEA), considerado técnica no paramétrica de análisis de eficiencia relativa. Los resultados apuntaron que los programas de postgrado fueron más eficientes en 2006, seguidos por 2004 y 2005 , respectivamente. Se ha notado también que, como promedio, los programas vinculados a las instituciones privadas de enseñanza fueron mas eficientes que los de la red pública en el trienio 2004/ 2006. Los gestores de esos programas pueden utilizar el análisis de la eficiencia relativa como estrategia de benchmarking, adoptando las mejores practicas observadas en los programas eficientes, buscando la maximización de la eficiencia en su gestión.

Palabras-clave: desempeño, Análisis Envoltorio de Datos.

Analysis of the relative efficiency of the academic postgraduation programs in Administration, Accounting and Tourism

Ney Paulo Moreira, Nina Rosa da Silveira Cunha, Marco Aurélio Marques Ferreira and Suely de Fátima

Ramos Silveira

This study was carried out to present an alternative analysis model of the efficiency of the postgraduation programs in Administration, Accounting and Tourism in the private and public graduation institutions. The study was theoretically based on both efficiency and optimization of resources, as taking the maximization of the return subjected to limitations of the resources as reference. The Data Envelopment Analysis (DEA) as nonparametric technique for the relative efficiency 
analysis was used as analytical model. According to the results, the postgraduation programs were more efficient in 2006, followed by 2004 and 2005, respectively. It was also observed that, on average, the programs of the private teaching institutions were more efficient than those in the public net during the three-year period (2004/2006). The managers of those programs can use the relative efficiency analysis as benchmarking strategy, by adopting the best practices observed in the efficient programs, in order to reach the maximization of the efficiency in their management.

Keywords: performance, Data Envelopment Analysis.

Ney Paulo Moreira

Bacharel em Ciências Contábeis, mestre em administração pela Universidade Federal de Viçosa (UFV). Professor assistente da (UFV), Campus Rio Paranaíba. Contato: ney.moreira@ufv.br

Nina Rosa da Silveira Cunha

Bacharela em Direito, (FUIT), mestre em Administração (UFMG) e doutora em Economia Aplicada (UFV). Professora associada do Departamento de Administração da Universidade Federal de Viçosa. Contato: ninarosa@ufv.br

Marco Aurélio Marques Ferreira

Bacharel em Administração, mestre em Economia Aplicada e doutor em Economia Aplicada pela Universidade Federal de Viçosa (UFV). Professor adjunto do Departamento de Administração da UFV. Contato: marcoaurelio@ufv.br Suely de Fátima Ramos Silveira

Bacharela em Ciências Econômicas e mestre em Economia Rural pela UFV; doutora em Economia Aplicada pela ESALQ/USP. Professora adjunta do Departamento de Administração da Universidade Federal de Viçosa (UFV). Contato:sramos@ufv.br 


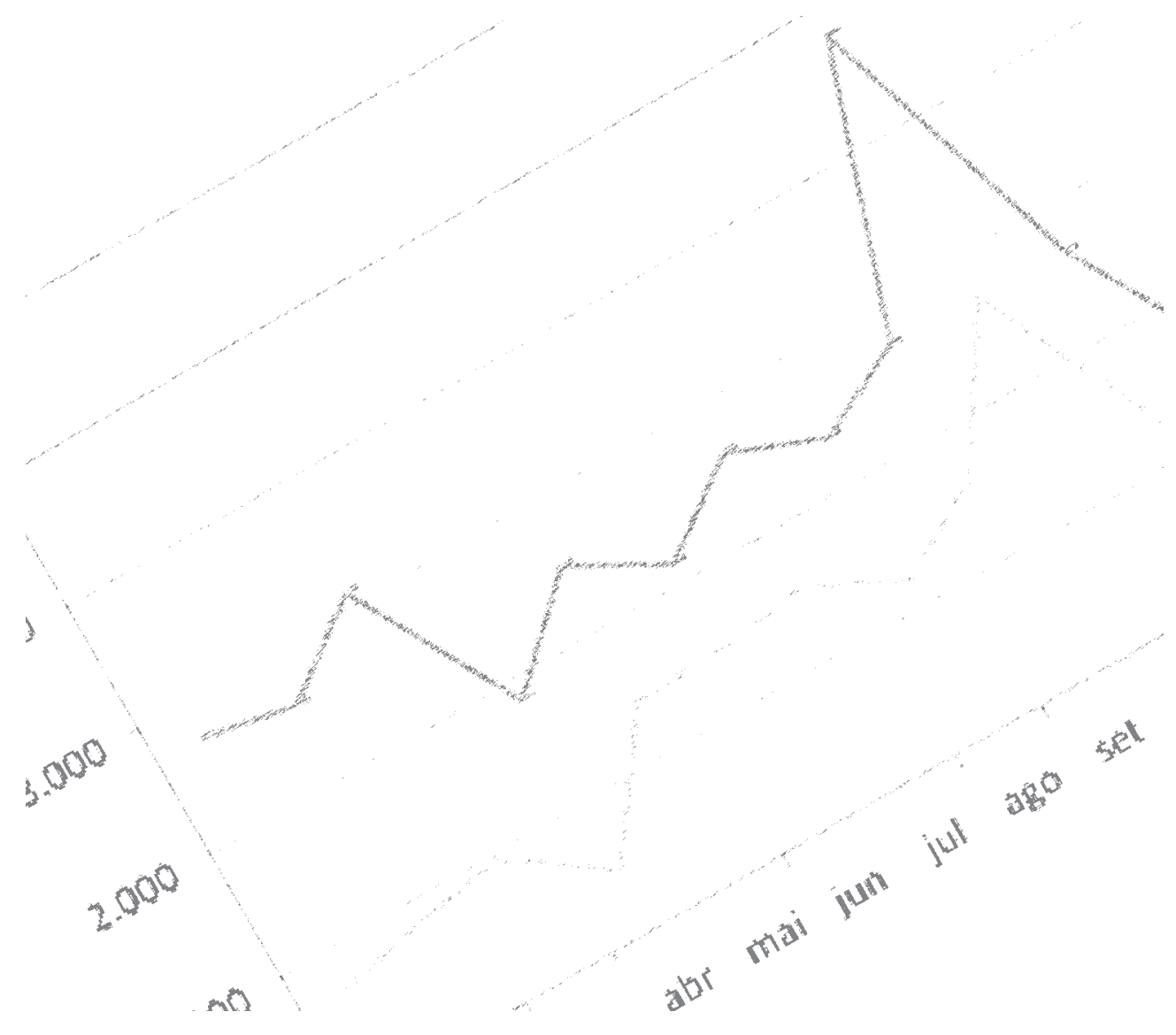

